\title{
COHOMOLOGIE LOCALE DE CERTAINS
} ANNEAUX AUSLANDER-GORENSTEIN

\author{
Marib-Paule Malliavin \\ Dédié à la mémoire de Pere Menal
}

\begin{abstract}
We give axiomalic conditions in order to calculate the local co homology of some idompotent keruel functors. The results lie on some new dimension introduced by T. Levasseu for AuslanderGorcnstein rings. Under some hypothesis, we generalize previous result.
\end{abstract}

Nous donnons ici des conditions aussi axiomatiques que possible pour calculer la cohomologie locale de certains foncteurs noyaux idempotents.

Les résultats reposent sur la découverte récente faite par ' $\mathrm{T}$. Levassenr d'me dimersion pour les anneaux appelés Auslander-Gorenstein et qui sont des généralisations naturelles des ameaux de Gorenstein commutatifs.

En imposant à cette dimension de satisfaire un certain nombre d'hypothèses on retrouve certains des résultats de [B.M] et [Ma2].

\section{Généralités}

Soit $A$ un ameit. Sauf mention du contraire, tous les $A$-modules sont des modules à gauche. On notera $\operatorname{Mod} A$ la catćgorie des $A$-rnodules à gauche et par $\operatorname{Mod}_{f} A$ la sous-catégorie des modules de type fini. On notera, lorsque cela sera nécessaire, par $\operatorname{Mod}^{\prime}(A)$ et $\operatorname{Mod}_{f}^{d}(A)$ les catégories correspondantes de $A$-modules à droite. On notera ${ }_{A} M$ ou $M_{A}$ pour indiquer que $M$ est un $A$-module à gauche ou à droite. Cette notation sera utiliséc surtout dans le cas où $M$ est un $A$-bimodule.

L'anneau $A$ est dit noethérien s'il est noethérien à gauche et à droite. Tous les anneaux qui interviendront seront noethériens. 
Soit ${ }_{A} M$ (resp. $M_{A}$ ) un $A$-module. Un élément $a \in A$ est dit non diviseur de zéro dans $M$ si $a x=0, x \in M$, entraine $x=0$ (resp. $x a=0$ entraîne $x=0$ ). Si $M$ est un bimodule et si $a \in A$ est non diviseur de zéro dans ${ }_{A} M$ (resp. $M_{A}$ ) on dit que $a$ est non diviseur de zéro à gauche (resp. à droitc) dans $M$. Si $a$ est non diviseur de zéro à gauche et à droite dans $A$ on dit que $a$ est non diviseur de zéro. Par exemple, si $A$ est noethérien et premier et si $I$ est un idéal bilatère de $A$ non nuI, $I$ contient un non diviseur de zéro.

Soit $M$ un $A$-module, on notera $d h_{A} M$ la dimension homologique de $M$ et inj. $\operatorname{dim}_{A} M$ la dimension injective de $M$. On dira que $A$ est $d e$ dimension homologique globale finie si la dimension homologique globale de ${ }_{A} A$ et celle de $A_{A}$ sont finies; elles sont alors égales car $A$ est noetherien $[\mathbf{A}]$. On notera alors gl $\operatorname{dim} A$ la dimension homologique globale de $A$. On dira que $A$ est de dimension injective finie si les modules ${ }_{A} A$ et $A_{A}$ sont de dimension injective finies; elles sont alors égales [Z], car $A$ est noetherien.

Si $M$ est un $A$-module à gauche, on appelie grade de $M$ et on note $j_{A}(M)$ (ou $j(M)$ si aucune confusion peut en résulter) le nombre cntier naturel ou $+\infty$ défini par:

$$
j_{A}(M)=\operatorname{Inf}\left\{i, \operatorname{Ext}_{A}^{i}(M, A) \neq 0\right\}
$$

On a évidemment $j_{A}((0))=+\infty$. Si $M$ est un $A$-module à droite on utilisera la notation $j_{A}^{d}(M)$ ou $j^{d}(M)$ pour le grade de $M$. On a toujours $j_{A}(M) \leq d h_{A}(M)$ pour $M \neq 0$ et si inj. $\operatorname{dim}(A)=\mu<\infty$ on a de façon evidente $j_{A}(M) \leq \mu$ pour tout $A$-module non nul $M$. On a les propriétés analogues pour $j_{A}^{d}$.

\section{Conditions d'Auslander. Anneau Auslander-Gorenstein et dimension}

Les définitions suivantes se trouvent dans [BJ2], [Bj-Ek], [Ek]; [Le2].

2.1. Définition. Soit $A$ un anneau noethérien. Un $A$-rnodule à droite ou à gauche de type fini $M$ satisfait la condition d'Auslander si quel que soit $q \geq 0$ on a $j_{A}(N) \geq q$ pour tout $A$-sous-module $N$ de type fini de $\operatorname{Ext}_{A}^{q}(M, A)$.

2.2. Définition. L'anneau noethérien est dit Auslander-Gorenstein (resp. Auslander régulier) de dimension $\mu$ si

1) inj. $\operatorname{dim} A=\mu<\infty$ (resp. gl $\operatorname{dim} A=\mu<\infty$ ),

2) chaque $A$-module à droite ou à gauche de type fini satisfait la condition d'Auslander. 
11 est clair qu'un anneau Auslander régulier cst Auslander-Gorenstein. Dans l'article [F.G.R.] les anneaux Auslander-Gorenstein de dimension injective $\mu$ sont appelés annenux $\mu$-Gorenstein. La condition d'Auslander dans l'article précédemninent cité est étudićc dans le contexte plus général de certaines catégories additives. D'autre part il résulte de [Ba] qu'un amneau commutatif nocthérien de dimension injective finie est AuslanderGorenstein, ceci n'est pas vrai dans le cas non commutatif comme le montre un exemple de I. Reiten [Re, Exemple 2.4.6].

Si $A$ est un amneau Auslander régulier (resp. Auslander-Gorenstein) et $S$ est un système de Ore ì droite et à gauche de $A$, alors l'anneau des fractions $S^{-1} A$ est aussi Auslander régulier (resp. Auslander-Gorenstcin); en effet si $M$ (resp. $N$ ) est un $A$-module à gaucke (resp. à droite) les $S^{-1} A$-modules à droite $\operatorname{Ext}_{A}^{i}(M, A) S^{-1}$ et $\operatorname{Ext}_{S^{-1}}^{i}\left(S^{-1} M, S^{-1} A\right)$ sont isomorphes ainsi que les $S^{-1} A$-modules à gauche $S^{-1} \operatorname{Ext}_{A}^{i}(N, A)$ et $\operatorname{Ext}_{S^{-1} A}^{i}\left(N S^{-1}, S^{-1} A\right)$.

Le plus sonvent les anneaux Auslander réguliers sont des anneaux positivement filtrés dont le gradué associć cst un anneau de polynômes. Tel est les cas de l'algèbre de Weyl d'indice $n$ sur un corps, ou de l'algèbre enveloppante d'une algèbre de Lie $g$ de dimension finie sur un corps. Plus généralement si $R$. est une $k$-algèbre qui est un anneau Auslander régulier (rcsp. Auskander-Gorenstein) et si $g$ est une $k$-algèbre de Lie de dimension finie opérart sur $R$ par $k$-dérivations, l'algèbre $R * U(g)$ (McRo] est elle-même Auslander régulic̀rc (resp. Auslander-Gorenstein).

2.3. Le résultat principal dans l'étude des anneaux de AuslanderCrorcnstein est contenu dans le résultat suivant dont la preuve peut être trouvée dans [Le1] et qui généralise des résultats antérieurs de [Bj1].

Théorème. Soit $A$ utn cunneau noethérien tel que $\mu=\operatorname{inj} \cdot \operatorname{dim} A<\infty$. Soit $M$ un élément de $\operatorname{Mod}_{f}(A)$.

(a) Il existe une suite spectrale convergente dars $\operatorname{Mod}_{f}(A)$, soit

$$
E_{2}^{p,-q}(M):=\operatorname{Ext}_{A}^{p}\left(\operatorname{Ext}_{A}^{q}(M, A), A\right) \Rightarrow H^{p-q}(M)
$$

où $H^{p-q}(M)=0$ si $p \neq q$ et $H^{0}(M)=M$. Il en résulte une filtration finie sur $M$, appelée la b-filtration qui est de la forme

$$
(0)=F^{\prime+1} M \subseteq F^{\prime} M \subseteq \cdots \subseteq F^{1} M \subseteq F^{0} M=M
$$

(b) si $A$ est Auslander-Gorenstein on a des suites exactes

$$
0 \longrightarrow \frac{F^{p} M}{F^{p+1} M} \longrightarrow E_{2}^{p,-p}(M) \longrightarrow Q(p) \longrightarrow 0
$$


où $p=0,1, \ldots, \mu$ et où $Q(p)$ est un sous-quotient de

$$
\underset{i \geq 1}{\mu-(p+1)} \operatorname{Ext}_{A}^{p+i+1}\left(\operatorname{Ext}_{A}^{p+i}(M, A), A\right)
$$

(c) les constructions précedentes sont fonctorielles en $M$.

Remarque. Si $M$ est un $A$-bimodule, on peut appliquer le théorème à ${ }_{A} M$ et d'après le point (c) il résulte que la b-filtration de ${ }_{A} M$ est formée de sous-bimodules de $M$, que les suites exactes établies en (b) sont des suites exactes de bimodules. Cependant on prendra garde que, par exemple, la $b$ filtration pour ${ }_{A} M$ n'a aucune raison de coïncider en général avec la $b$-filtration pour $M_{A}$. De même les modules $Q(p)$ apparaissant pour ${ }_{A} M$ ne sont pas nécessairement identiques à ceux apparaissant pour $M_{A}$. On pourrait aussi déduire la remarque précédente d'une étude détaillée de la preuve du théorème 2.3 .

2.4. Le théorène suivant est le résultat 1.8 de $[\mathbf{B j} \mathbf{2}]$ :

Théorème. Soit $A$ un anneau Auslander-Gorenstein. Si $0 \rightarrow M^{\prime} \rightarrow$ $M \rightarrow M^{\prime \prime} \rightarrow 0$ est une suite exacte de $A$-module de type fini à gauche on a $j_{A}(M)=\operatorname{Inf}\left\{j_{A}\left(M^{\prime}\right), j_{A}\left(M^{\prime \prime}\right)\right\}$ et le même résultat est vrai pout une suite exacte de A-modules à droite.

2.5. Par analogie avec certaines propriétés des dimensions, J.E. Björk introduit [Bj1] la défnition suivante:

Définition. Soient $A$ un amneau noethérien et $M$ un $A$-module à gauche de type fini non nul. Le module $M$ est dit pur si l'on a $j_{A}(N)=$ $j_{A}(M)$ pour tous les sous-modules non nuls $N$ de $M$. Lorsque $d=j_{A}(M)$ on précise en disant que $M$ est $d$-pur.

On a alors le théorème suivant dont on pourra trouver la preuve dans $[\mathbf{B j 2}]$, [Le1], [Bj-Ek] et [Li-Hu]:

Théorème. Soient $A$ un anneau Auslander-Gorenstein, $M$ un $A$ module de type fini non nul, $d$ le grade de $M$. Alors

(a) $\operatorname{Ext}_{A}^{d}(M, A)$ est d-pur (la démonstration de ce fait est dûe à $R$. Fossum); en particulier $\operatorname{Ext}_{A}^{d}\left(\operatorname{Ext}_{A}^{d}(M, A), A\right) \neq 0$.

(b) Si $\operatorname{Ext}_{A}^{p}\left(\operatorname{Ext}_{A}^{p}(M, A), A\right)$ est non nul, c'est un module pur.

(c) Le module $M$ est pur si el seulement si $\operatorname{Ext}_{A}^{p}\left(\operatorname{Ext}_{A}^{p}(M, A), A\right)=0$ pour chaque $p \neq d$.

(d) $F^{p} M$ est le plus grand sous-module $X$ de $M$ tel que le grade de $X$ est $\geq p$.

(e) $d=\sup \left\{p, M=F^{p} M\right\}$. 
Remarque. On peut démontrer directement à partir du théorème 2.5 (d) que si $M$ est un bimodule, $F^{p} M$ est, pour tout $p$, ur sous-bimodule de $M$. En effet soit, $a \in A$; alors $\left(F^{p} M\right) a$ est image homomorphe du $A$-module à gauche $F^{p} M$; donc d'après $2.4, j_{A}\left(F^{p} M\right)$ est inférieur à. $j_{A}\left[\left(F^{p} M\right) a\right]$. Par le théorène précédent, il vient $\left(F^{p} M\right) a \subseteq F^{p} M$.

2.6. On trouvera dans [Le2, 'Thm. 4.2] la preuve du thcorème suivant:

Théorème. Soient $A$ un anneau Austander-Gorenstein et $M \in$ $\operatorname{Mod}_{f}(A)$ avec $j_{A}(M) \geq n$. Soit $\left(M_{i}\right)_{i \in \mathbb{N}}$ une suite décroissunte de sousmodules de $M$. Alors il existe $q \in \mathbb{N}$ tel que $j_{A}\left(M_{i} / M_{\imath+1}\right) \geq n+1$ pour tous les $i$ tels que $i \geq q$.

Corollaire. Si $M$ est un module à ganuche de type fini sur un anneau Auslander-Gorenstein $A$ et si $\varphi \in \operatorname{End}_{A}(M)$ est injectif alors $j_{A}\left(M / \varphi(M) \geq j_{A}(M)+1\right.$.

Preuve: On considère la suite décroissantie de sous-modules $\left\{\varphi^{i}(M)\right\}_{i \in \mathbb{N}}$ el on a pour tout $i$ un isomorphisme civident $\frac{\varphi^{2}(M)}{\varphi^{i+1}(M)} \rightarrow \frac{\varphi^{i+1}(M)}{\varphi^{i+2}(M)}$ : puisque $\varphi$ est injectif́. Donc d'après le théorème $j\left(\frac{M}{\varphi(M)}\right) \geq j(M)+1$.

Ce corollaire est démontré de façon plus générale en 4.3 de [Le2].

2.7. On trouvera dans [Le2] la définition et la prenve de la proposition suivante:

Définition. Soit $A$ un anreau Auslander-Gorenstein de dimension $\mu$. On définit la dimension de $M \in \operatorname{Mod}_{f} A$ par $\delta(M)=\mu-j(M)$. Puisque $j(M)=0,1, \ldots, k$ ou $\infty$, on a $\delta(M)=0,1, \ldots, \mu$ oll $-\infty$.

Proposition. La forction $\delta: \operatorname{Mod}_{f} A \rightarrow\left\{0, \ldots, \mu_{,}, \infty\right\}$ possède les propriétés suivantes:

(i) $\delta(0)=-\infty$.

(ii) Si $0 \rightarrow M^{\prime} \rightarrow M \rightarrow M^{\prime \prime} \rightarrow 0$ est une sutite de A-modules à gauche de type fini, on a $\delta(M)=\sup \left\{\delta\left(M^{\prime}\right), \delta\left(M^{\prime \prime}\right)\right\}$.

(iii) Si $P$ est un idéal premier de $A$ et $M$ est un $A / P$-module à gauche de type fini el de torsion, alors $\delta(N) \leq \delta(A / P)-1$.

(iv) Si $M=M_{0} \supset M_{1} \supset \cdots \supset M_{i} \supset \ldots$ est une chaîne de sousmodules, alors $\delta\left(M_{i} / M_{i+1}\right) \leq \delta(M)-1$ pour $i$ suffisamment grand.

Ces propriétés ressemblent fort à la définition albstraite donnće par A. Joseph (Application de la théorie des anneaux aux algèbres enveloppartes, Cours de 3e cycle, 1981, Paris VI) à l'exception de deux propriétés sur les queles nous reviendrons plus bard, à savoir que $\delta(M)=$ 
$-\infty$ si et seulement si $M=0$ et $\delta(M)=0$ si el seulement si $M$ est un $A$-module non nul de longucur finie. Les propriétés (i), (ii), (iii), (iv) et les denx propriétés supplémentaires sont vérifices par la dimension de Krull et la dimerision de Gelfand-Kirillov (pour les algèbres sur des corps), lorsque cette dernière est partitive.

Si $A$ est un anneau commutatif Gorenstein de pure dimcnsion, $\delta$ est la dimension de Krull clussique de $A$.

Si $A$ est l'algèbre enveloppante d'une algèbre de Lie résoluble de dimension finie sur un corps $k$ de caractéristique nulle, $\delta$ cst la dinnension de Gelfand-Kirillov sur $k$.

Définition. On à une définition analogute à la précédente pour les modules à droite de type fini que l'on notera pour la différencier $\delta^{d}$.

Remarque. A l'aide de $\delta$, le résultat du théorème 2.3 et 2.5 peut être interprété comme suit. Posons $d=j_{A}(M), \delta(M)=s=\mu-d$. Le sous-module $F^{p} M$ est le plus grand sous-module $X$ de $M$ tel que $\delta(X) \leq \mu-p$. Le module $\operatorname{Ext}_{A}^{d}(M, A)$ est $\mu-s$ pur. On a des suites exactes fonctorielles

$$
0 \longrightarrow \frac{F^{p} M}{F^{p+1} \bar{M}} \longrightarrow \operatorname{Ext}_{A}^{p}\left(\operatorname{Ext}_{A}^{p}(M, A), A\right) \longrightarrow Q(p) \longrightarrow 0
$$

où $\operatorname{Ext}_{A}^{p}\left(\operatorname{Ext}_{A}^{p}(M, A), A\right)$ est soit nul soit $p$-pur et $\delta(Q(p)) \leq \mu-p-2$.

2.8. Définition. Un $A$-module $M \in \operatorname{Mod}^{f} A$ non nul est dit critique si l'on a $\delta(M / N) \lesseqgtr \delta(M)$ pour chaque sous-module non nul $N$ de $M$. Si $\delta(M)=s$ on dit alors que $M$ est $s$-critique et ccci est équivalent à $j(M / N)>j_{A}(M)=\mu-s$ pour tout sous-module non nul $N$ de $M$.

Proposition [Mc-Ro, 6.2.10]. Tout module non nul de type fini $M$ possède un sous-module critique.

Preuve: On raisonne par l'absurde et l'on suppose qu'il existe un cntier $n \geq 0$ et un module $M, \delta(M)=n$, n'ndmettant pas de sous-module critique. D'après la propriété 2.7 (ii) on peut trouver une chaine strictement décroissante $M=M_{0} \supsetneqq M_{1} \supsetneq \ldots$ de sous-modules de $M$ tels que $\delta(M)=\delta\left(M_{i}\right)=\delta\left(M_{i} / M_{i+1}\right)$ ce qui contredit, $2.7(\mathrm{iv})$.

2.9. Le résultat suivant est bien connu pour la dimension de GelfandKirillov. La preuve est la même ici.

Proposition. Supposons la dimension $\delta$ idéal-invariante, c'est-ia-dire que pour tout $A$-module de type fini $M$ ct tout idéal bilatère $I$ de $A$, on a $\delta\left(I \otimes_{A} M\right) \leq \delta(M)$. Alors si $M$ est un module criticque, son annulateur est premier. 
Prenve: Soit $P=\operatorname{Ann}_{A}(M)$ et soit $I$ et $J$ deux idéaux bilatères de $A$ tels que $I J \subseteq P$. De l'application surjective naturelle $I \otimes_{A} M / J M \rightarrow I M$ résulte par 2.7 (ii) que $\delta(I M) \leq \delta\left(I \otimes_{A} \frac{M}{J M}\right)$. Puis par idéal-invariance $\delta\left(I \otimes_{A} \frac{M}{J M}\right) \leq \delta\left(\frac{M}{J M}\right)$. Donc $\delta(I M) \leq \delta\left(\frac{M}{N M}\right)$. Cormme $M$ est critiquc, ou bien $J M=0 \mathrm{et}, J \subseteq P$ ou bien $J M \neq 0$. Donc $\delta(M / J M) \lessgtr \delta(M)$ et $\delta(I M) \lessgtr \delta(M)$. Donc par $2.7(\mathrm{ii}) \delta(M)=\delta\left(\frac{M}{M}\right)$ et douc $I M=0$. D'où $I \subseteq P$.

\section{La résolution injective minimale de certains anneaux Auslander-Gorenstein}

Dans toute la suite on considèrera des idéaux bilatères $P$ de $A$ et des bimodules correspondants $M=A / P$. Pour le calcul de $\operatorname{Ext}_{A}^{i}(M, A)$ on utilisera la structure de $A$-module à gauche $\operatorname{sur} M$ et on mettra sur $\operatorname{Ext}_{A}^{i}(M, A)$ la structure de $A / P$ - $A$-binodule, $A$-module à droite provenant de la structure $A_{A}$ et $\frac{A}{P}$-module ì gatuche provenant $M_{A / P}$. On calculera $\operatorname{Ext}_{A}^{j}\left(\operatorname{Ext}_{A}^{i}(M, A), A\right)$ pour la structure de $A$-module à droite de $\operatorname{Ext}_{A}^{i}(M, A)$.

3.1. Lemme. Soient $A$ un anneau Auslander-Gorenstein de dimension injective $\mu, P$ un inderl premier de $A, M=A / P$ et $j(M)=d$. Alors on a $M=F^{d} M$ et $F^{d+1} M=(0)$; en particulier $M$ est $d$-pur.

Preuve: D'après 2.5(c) on a $M=F^{d} M$ et d'après $2.5(a) \operatorname{Ext}_{A}^{d}$ $\left(\operatorname{Ext}_{A}^{d}(M, A), A\right)$ est non nul; il est donc $d$-pur. On en déduit que $j\left(F^{d} M / F^{d+1} M\right)=d=j(M)$. Si $F^{d+1}(M)$ n'était pas nul, il existerait un idéal bilatère $J$ de $A$ contenant stricternent $P$ tel que $F^{d+1} M=I / P$. L'idéal bilatère $I / P$ de l'anneau noethérien $A / P$ contiendrait un non diviseur de zéro. Donc $\delta(A / I) \leq \delta(A / P)-1 \lesseqgtr \delta(A / P)$. On obtiendrait la contradiction

$$
\delta\left(\frac{M}{F^{d+1} M}\right)=\delta\left(\frac{F^{d t} M}{F^{d+1} M}\right) \lessgtr \delta(M)=\delta(A / P) .
$$

3.2. Les deux théorèmes suivants sont essentiellement démontrés dans [Ma1]:

Théorème. Soit $A$ un anneau Auslander-Gorenstein de dimension injective $\mu$. On suppose la dimension $\delta$ idéal-invariante. Soit $P$ un idéal completement premier de $A, M=A / P$ et $j_{A}(M)=d$. Soit $F r(A / P)$ le 
corps des fractions de $A / P$. Alors le $\operatorname{Fr}(A / P)$ espace vectoriel à gauche $F r(A / P) \otimes_{A} \operatorname{Ext}_{A}^{d}\left(\operatorname{Ext}_{A}^{d}(M, A), A\right)$ est de dimension 1 .

Preuve: Considérons la suite exacte de A-bimodules

$$
0 \longrightarrow M \longrightarrow \operatorname{Ext}_{A}^{d}\left(\operatorname{Ext}_{A}^{d}(M, A), A\right) \longrightarrow Q(d) \longrightarrow 0
$$

où $\delta(Q(d)) \leq \delta(M)-2$ d'après la remarque 2.7. Daprès la propriêté (ii) de 2.7 , on a, en posant $\overline{Q(d)}=\frac{Q(d)}{P Q(d)}=\frac{A}{P} \otimes_{A} Q(d), \delta(\overline{Q(d)}) \leq \delta(Q(d)) \lesseqgtr$ $\delta(A / P)$. 11 résulte de $2.7($ ii) que cliaque sous-module à gauche monogène de $\overline{Q(d)}$ est de la forme $A / I$ où $I$ est un idéal à gauche de $A$ contenant strictement $P$. On a donc en posant $S=(A / P) \backslash\{0\}, S^{\cdot} \overline{Q(d)}=(0)$. Posons $V_{t}=\operatorname{Ext}_{A}^{d}\left(\operatorname{Ext}_{A}^{d}(M, A), A\right)$ et tensorisons la suite exacte de $A$ modules à gauche

$$
0 \longrightarrow M \longrightarrow V_{d} \longrightarrow Q(d) \longrightarrow 0 \text { раг } A / P .
$$

On obtient la suite exacte de $A / P$-modules à gauche

$$
\operatorname{Tor}_{1}^{A}(A / P, Q(d)) \longrightarrow A / P \longrightarrow \bar{V}_{d} \longrightarrow \overline{Q(d)} \longrightarrow 0
$$

oì on a posé $\bar{V}_{d}=V_{d} / P V_{d}$.

On décompose la suite précédente en les trois suites exactes

$$
\begin{aligned}
& \operatorname{Tor}_{1}^{A}(A / P, Q(d)) \longrightarrow J / P \longrightarrow 0 \\
& 0 \longrightarrow J / P \longrightarrow A / P \longrightarrow A / J \longrightarrow 0 \\
& 0 \longrightarrow A / J \longrightarrow \bar{V}_{d} \longrightarrow \overline{Q(d)} \longrightarrow 0
\end{aligned}
$$

où $J$ est un idćal à gauche de $A$ conterrant $P$.

Montrons que $J$ coincide avec $P$. En effet si l'on avait $J \supsetneqq P$, il résulterait de 2.7 (iii) que

$$
\delta(A / J) \leq \delta(A / P)-1 \varsubsetneqq \delta(A / P) .
$$

Tensorisant la suite exacte de $A$-modules à droite

$$
0 \longrightarrow P \longrightarrow A \longrightarrow A / P \longrightarrow 0
$$

par $Q(d)$, on obtient

$$
0 \longrightarrow \operatorname{Tor}_{1}^{A}(A / P ; Q(d)) \longrightarrow P \otimes_{A} Q(d) \longrightarrow Q(d) \rightarrow \overline{Q(d)} \longrightarrow 0
$$


LOCAI, COHOMOLOGY OF SOME AUSLANDER-GORENSTEIN RINGS 733

D'après 2.7 (ii) on a

$$
\delta\left(\operatorname{Tor}_{1}^{A}(A / P, Q(d)) \leq \delta\left(P \otimes_{A} Q(d)\right)\right.
$$

et en raison de l'idćal-invariance de $\delta$; on a

$$
\delta\left(P \otimes_{A} Q(d)\right) \leq \delta(Q(d)) \lessgtr \delta(A / P)
$$

D'après la suite exacte (1) et en appliquant $2.7($ ii) on a

$$
\delta(J / P) \leq \delta\left(\operatorname{Tor}_{i}^{A}(A / P, Q(d)) .\right.
$$

Il résulte de tout ceci qué $\delta(J / P) \supsetneqq \delta(A / P)$.

La suite exacte (2) et lá partitivité de $\delta$ (cí. 2.7 (ii)) donne

$$
\delta(A / P)=\sup (\delta(J / P), \delta(A / J))
$$

En utilisant les inégalités $\delta(A / J) \leqq \delta(A / P)$ et $\delta(J / P) \leqq \delta(A / P)$ on arrive is une contradiction.

On a donc démontré que $j=P$. La suite exacte (3) devient

$$
0 \longrightarrow A / P \longrightarrow \bar{V}_{d} \longrightarrow \overline{Q(d)} \longrightarrow 0 .
$$

Eu appliquant $S^{-1}$ à cette suite, où $S=(A, P) \backslash\{0\}$, on obtient

$$
0 \longrightarrow \operatorname{Fr}(A / P) \longrightarrow S^{-1} \bar{V}_{d} \longrightarrow S^{-1} \overline{Q(d)} \longrightarrow 0 .
$$

Cornme $S^{-1} \overline{Q(d)}=0$, il vient

$$
\begin{aligned}
F r(A / P) \equiv S^{-1} \bar{V}_{d} & =F r\left(\frac{A}{P}\right) \otimes_{A} V_{d}= \\
& =F r(A / P) \otimes_{A} \operatorname{Ext}_{A}^{d}\left(\operatorname{Ext}_{A}^{d}(M, A), A\right)
\end{aligned}
$$

qui est l'isomorphisme cherché.

3.3. On va étendre la dimension $\delta_{g}$ à tout $A$-module à gauche non nécessairement de type fini en posant

$$
\delta_{g}(N)=\sup \left\{\delta_{g}(Q)\right\}
$$

oì $Q$ parcourt les sous-modules de type fini de $N$. Il est clair d'après 2.7 (ii) que $\delta_{g}(N)=\delta(N)$ si $N$ est de type fini. 
Définition. On dira qu'un idéal premier $P$ de $A$ est $d$-modéré $a$ gauche si pour $d=j(A / P)$ on a pour tout $i>d$,

$$
\delta_{g}\left(\operatorname{Ext}_{A}^{i}(A / P, A)\right) \supsetneqq \delta(A / P) .
$$

11 est clár que si $\mu=d$, tout idéal premier de $A$ est $\mu$-modéré à gauche car alors pour $i>\mu$

$$
\operatorname{Ext}_{A}^{i}(A / P, A)=0 \text { donc } \delta_{g}\left(\operatorname{Ext}_{A}^{i}(A / P, A)\right)=-\infty \text {. }
$$

Remarque. Si $A$ est une algèbre sur un corps $k$ : il résulte de [Len, Lemme 2] que si $\delta$ est la $G K$-dimension de $A$, alors tout idéal premier de $A$ est modéré à gauche.

3.4. Définition. Soient $A$ un anneau noethérien, $P$ un idéal complètement premier de $A$. On notera $\mu_{i}(P, A)$ ct on appellera $i^{\text {icme }}$ invariant de Bass de $A$ la dimension sur le corps gauche $\operatorname{Fr}(A / P)$ de l'espace vectoriel

$$
F r(A / P) \otimes_{A / P} \operatorname{Ext}_{A}^{i}(A / P, A)
$$

Théorème. Soient $A$ un anneau Auslander-Gorenstein de dimensiont injective $\mu, P$ un idéal complètement premier de $A, d$ le grade du $A$ module à gauche $M:=A / P$. On a alors

(i) $\mu_{t}(P, A) \neq 0$ et $\mu_{i}(P, A)=0$ si $i \supsetneqq d$,

(ii) Si $P$ est d-modéré à gauche on a $\mu_{i}(P, A)=0$ pour $i \neq d$.

Preuve: Posons $S=(A / P) \backslash\{0\}, K=\operatorname{Fr}(A / P)$.

(i) On a évidemment $\mu_{i}(P, A)=0$ si $i<d$ puisque $\operatorname{Ext}_{A}^{i}(A / P, A)=0$ pour $i<d$. Montrons que $\mu_{d}(P, A) \neq 0$. Pour cela raisonnons par l'absurde; supposons que $\mu_{d}(P, A)=0$ et arrivons à une contradiction

$$
K \otimes_{A} \operatorname{Ext}_{A}^{d}(M, A)=S^{-1} \operatorname{Ext}_{A}^{d}(M, A)=0 .
$$

En tant que $A$-module à droite, le bimodule $\operatorname{Ext}_{A}^{d}(M, A)$ est de type fini; donc il existe $s \in A \backslash P$ tel que $s \operatorname{Ext}_{A}^{d}(M, A)=0$. La multiplication à gauche par $s$ dans $\operatorname{Ext}_{A}^{d}(M, A)$ provient de la multiplication à droite par $s$ dans $M=A / P$. Mais $P$ étant complètement premier, la multiplication à droite par $s$ dans $A / P$ conduit à une suite exacte de $A$-modules à gauche.

$$
0 \longrightarrow A / P \stackrel{s}{\longrightarrow} A / P \longrightarrow \frac{A}{P+A s} \longrightarrow 0
$$


d'où la suite exacte de $A$-modules à droite

$$
\operatorname{Ext}_{A}^{d}(A / P+A s, A) \rightarrow \operatorname{Ext}_{A}^{d t}(A / P, A) \stackrel{s}{\longrightarrow} \operatorname{Ext}_{A}^{d t}(A / P, A) .
$$

D'après $2.7($ iii $)$ on a. $\delta(A / P+A s) \lessgtr \delta(A / P)=\mu-d$. Donc $\operatorname{Lxt}_{A}^{d}(A / P+A s, A)=0$ et ha multiplication à ganche par s est injective; comme $s \operatorname{Ext}_{A}^{d}(A / P, A)=0$, on a $\operatorname{Ext}_{A}^{d}(A / P, A)=0$, ce qui contredit. la définition de $d$.

(ii) Supposons $P$ modérć à gauche et $i>d$ et notons $N_{i}$ le $\frac{A}{i}-A$ bimodule $\operatorname{Ext}_{A}^{i}(M, A)$. Alors $\delta_{g}\left(N_{i}\right) \leqq \delta(A / P)$. Par suite chaque sousmodule monogène $Q$ de $A / P\left(N_{i}\right)$ est, tel que $\delta(Q) \lessgtr \delta(A / P)$. Comme $Q$ est isomorphe à $A / I$ où $I$ est un icléal à gauche de $A$ contenant $P$, cet. idéal contient strictement $P$. Dons, puisque $P$ est complètement premier, $S^{-1} Q=0$. Il en résulte que $S^{-1} N_{i}=(0)$ c'est-à-diro $\mu_{i}(P, A)=0$.

Remarques. 1) Pour m irléal complètement premier, la condition de modération à gauche est équivaleute au fait que $\mu_{i}(P, A)=0$ pour $i \neq d$ où $d=j(A / P)$. En clfet si $d<i$ et $S^{-1} \operatorname{Ext}_{A}^{i}(A / P, A)=0$, alors pour tout sous-module monogene $Q$ du $A / P$-module à gauche $\operatorname{Ext}_{A}^{i}(A / P, A)$ on a $S^{-1} Q=0$. Donc $Q \simeq A / I$ oì $I$ est un idéal à gatuche de $A$ contenant strictement $P$. Par 2.7(ii), ol a $\delta_{g}(Q)<d$ et donc $\delta_{g}\left(\operatorname{Ext}_{A}^{i}(A / P, A)\right)<$ $\delta(A / P)$.

2) Si $A$ est le localisé en une clique d'unc algèbre enveloppante d'une algèbre de Lic résoluble de dimension finie alors $\mu(A)$ est la dimension de Krull de $A$ (cf. $[\mathrm{Br}])$ et tout idéal (maximal) de $A$ est modéré à gauche pour la dimension $K$-dim $A-j(M)$.Il ne devrait pas etre difficile de démontrer que pour tout $A$-module à geuche $M$ de type fini de $A$ on a $K-\operatorname{dim} M=K-\operatorname{dim} A-j(M)$ mais ceci est vrai pour un module de la forme $A / P$ où $P$ est un idéal maximal et, par récurrence sur la longueur, on le démontre pour tout $A$-module de longueur finie.

3.5. On supposera que l'anneau $A$ est Auslander-Gorenstein, que la dimension $\delta$ est icléal-invariante que tous les idérux premicrs de $A$ sont complètement premiers et nodérés à gauche. Comme l'anneau $A$ est noethérien: chaque $A$-modulc (à gauche) injectif est somme directe d'injectifs indécomposables ([Str., V.4.5]). Un $A$-module injectif inclécomposable est de la forme $E(X)$, l'enveloppe injective d'un $A$ module $\delta$-criticue $X$ où $\operatorname{Am}_{g}(X)=P$ est premier; ceci résulte du fait que les injectifs indécomposables sont uniformes et que chaque module non nul contient un sous-module $\delta$-critique dont l'anmulateur est premier, car $\delta$ est supposé idéal-invariante. Alors ou bien le module $X$ est sans torsion comme $A / P$-nodule anquel cas $X$ est isomorphe à un idéal à gauche du domaine $A / P$, puisque $P$ est supposé complètement premier et l'on a $E(X)=E(A / P)$; ou bien $X$ est un $A / P$-modulc de torsion. 
Finalement sous les hypothèses précédentes, tout $A$-module injectif est de la forme $E^{I} \oplus E^{I I}$ où $E^{I}=\underset{P \in \operatorname{Spcc}(A)}{\oplus} \nu(P) E(A / P)$, les $\nu(P)$ étant des cardinaux et où $E^{I I}$ est l'enveloppe injective d'une somme de modules $\delta$-critiques chacun d'eux étant de torsion modulo son annulateur.

En particulier ceci s'applique aux termes $E_{i}(i=0,1, \ldots, \mu)$ de la résolution injective minimale de $A A$ et il est clair que $\mu_{i}(P, A)$ est le cardinal $\nu(P)$ défini dans la décomposition de $E_{i}^{I}$ en facteur irréductibles; en effet puisque $\operatorname{Hom}_{A}(A / P, \oplus \nu(P) A / P) \simeq \oplus \nu(P) A / P$, isomorphisme de $A / P$-modules à gauche, il résulte de la définition des groupes $\operatorname{Ext}_{A}(-, A)$ en terme de la résolution injective de ${ }_{A} A$, que $\mu_{i}(P, A)$ est exactement le rang uniforme maximal d'un $A / P$-sous-module sans torsion du module à gauche $\operatorname{Ext}_{A}^{i}(A / P, A)$. On a donc la généralisation suivante d'un résultat de $[\mathrm{Ma1}]$.

Théorème. Soient $A$ un anneau Auslander-Gorenstein, dont la dimension $\delta$ est idéal-invariante et dont tous les idéaux premiers sont complètement premiers et modérés à gauche. Alors la résolution injective minimale du A-module ${ }_{A} A$ est de la forme

$$
0 \longrightarrow A \longrightarrow E_{0} \longrightarrow E_{1} \longrightarrow \cdots \longrightarrow E_{\mu} \longrightarrow 0
$$

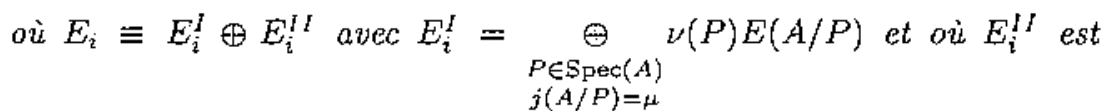

l'enveloppe injective d'une somme de modules $\delta$-critiques, chacun d'eux étant de torsion modulo son annulateur.

3.6. Dans ce paragraphe nous utiliserons une forme affaiblie du théorème 3.5 et qui se démontre de marière analogue.

Théorème. Soient $A$ un anneau Auslander-Gorenstein de dimension injective $\mu$ dont tout idéal premier de grade $\mu$ est completement premier. On suppose que la dimension $\delta$ est idéal-invariante. Alors le dernier temre de la résolution injective minimale de ${ }_{A} A$ est $E_{\mu}=E_{\mu}^{I} \oplus E_{j t}^{I I}$ où $E_{\mu}^{I}=\underset{P \in \mathrm{Spec}(A)}{\oplus} \nu(P) E(A / P)$ et où $E_{\mu}^{I I}$ est l'enveloppe injective d'une $j(\Lambda / P)=\mu$

sonntne de modules $\delta$-critiques, chacun d'eux étant de torsion modulo son annulateur.

3.7. Les paragraphes 3.5 et 3.6 ne donnent aucune information sur les invariants de Bass $\nu(P)$. Cependant il existe des cas où l'on sait que $\nu(P)=1$ : c'est en premier lien celui des anneaux commutatifs Gorenstein [Ba] et en second celui des algèbres enveloppantes des algèbres de 
Lie résolubles de dimension finic sur un corps de caractéristique uulle [Ma1]. Ce demier résultal a été retrouvé par une autre démonstration utilisant la localisation en des cliques [Br-Le].

\section{Cohomologie locale de certains anneaux Auslander-Gorenstein}

4.1. Dans tout ce paragraphe $A$ designera un anneau noethérien sur lequel nous ferons les hypothèses (A), (B) ct (C) suivantes.

(A) Tout idéal à gnuche maximal de A est bilatère (et évidemment maximal). Un calcul évidert montre alors que tont ictéal maximal cst complètement premier.

(B) La famille $\mathcal{F}$ des idéaux bilatères co-artiniens de $A$ est une famille d'Artin-Rees à gauche, un idéal $I$ co-artinien étant tel que l'armeau $A / I$ est artinien. Il n'est pas nécessaire de préciser si l'idéal est co-artinien à droite ou à gauche puisque $A$ est noethérien à droite et à gauche.

Remarquons que $\mathcal{F}$ est la famille des idéaux bilatères de $A$ qui contiennent un produit d'idéaux bilatères maximaux. Rappclons qu'une farnille d'idéaux bilatères est dite d' $\Lambda$ rtin-Rees à gauche si elle vérific:

a) Si $I \in \mathcal{F}$ et $I \subseteq J$ oì $J$ est un idéal bilatère de $A$, alors $J \in \mathcal{F}$.

b) Si $I$ et $J \in \mathcal{F}$ alors le produit $I J$ appartient à $\mathcal{F}$.

c) Si $N$ est un $A$-nodule à gauche de type fini et $M$ un sous- $A$ module de $N$, si $I \in \mathcal{F}$, il existe $J \in \mathcal{F}$ tel que $J N \cap M \subseteq I M$.

Jes propriétés (A) et (B) sont vérifiées si $A$ esti l'algc̀bre enveloppante d'une algèbre de Lie résoluble de dinnension finie sur un corps de caractéristique nulle $[$ B.M $\}$ ou si $A$ est le localisé d'une telle algèbre on une clique [Ma2].

On notera $\sigma$ le foncteur noyau idempotent symétrique correspondant: à la farrille $\mathcal{F}$, c'est-ì-dire $\sigma=\underset{\mathcal{F}}{\lim _{\rightarrow}} \operatorname{Hom} A(A / I,-)$. Il résulte de [He-Ve, Proposition 2] et de [Ve, Proposition 5.8 que $\sigma$ est stable, c'est-ì-dire que la classe $\Pi_{\sigma}$ des $A$-modules de $\sigma$-torsion est fermée par enveloppe injective.

On notera $\operatorname{Mod}_{A}^{\mathcal{F}}$ la sous-catégorie pleine et épaisse de $\bmod _{A}^{f}$ dont les objets sont les $A$-modules dont l'annulateur appartient à $\mathcal{F}$.

La dernière hypothèse que l'on mettra sur l'anneau $A$ est la suivante:

(C) L'annean A est Austander-Gorenstein, $\delta$ est idéal-inuariunle; on a $\delta(M)=0$ si et seulement si $M$ est de longueur finic non nul; si $\delta(M)=-\infty$ alors $M=(0)$; cette dernière condition est trivialcment vérifiée si $A$ est Auslander régulier. 
4.2. On démontre comme dans la proposition 2.11 de [B.M] le résultat suivant:

Proposition. Soit $A$ un anneau vérifiant les conditions de 4.1 et soit 4 la dimension injective de $A$. On pose $T=\operatorname{Ext}_{A}^{\prime \prime}(-, A)$. Alors $T$ est naturellement équivalent sur la catégorie $\operatorname{Mod}_{A}^{\mathcal{F}} \dot{a} \operatorname{Hom}_{A}(-, E)$ oì $E=\underset{\mathcal{F}}{\lim _{\mathcal{H}}} \operatorname{Ext}_{A}^{\mu}(A / I, A)$. De plats $E$ est un $A$-module à gauche injectif.

Si on note $H_{\sigma}^{*}$ la cohomologie locule relative à $\sigma$, on a donc $H_{\sigma}^{\mu}(A)=F$.

On veut montrer que sous les hypothèses de $4.1, E$ apparâit comme le dernier terme de la résolution injective minimale du $A$-module à gauche ${ }_{A} A$.

4.3. Lemme. Soit I un idéal bilatère co-artinien de A. Alors on a

$$
\operatorname{Hom}_{A}\left(A / I, E_{q}^{I I}\right)=0 \text { pour tout } q=0, \ldots, \mu \text {. }
$$

Preuve: Il suffit de vérifier que $\operatorname{Hom}_{A}(A / I, H)=(0)$ pour tout facteur indécomposable $H$ de $E_{q}^{11}$. Raisonnons par l'absurde el supposons que $x$ soit un élément non nul de $H$ annulé par $I$. Alors $A x \subset H=E(A x)$ et $A x \simeq A / J$, où $J$ est un idéal à gatuche de $A$ contenant $I$ donc co-artinierl. Quitte à remplacer $A x$ par un sous-module simple quil contient, on peut supposer l'idéal J maximal à gauche. Done par la condition (A), J est bilatère et on a $H \simeq E(A / J)$ oì $J$ est un idéal maximal bilatère ce qui contredit le fait que $H \subseteq E_{q}^{H}$.

Corollaire. Si J est un idéal à gauche co-artinien de $A$, on a

$$
\operatorname{Hom}_{A}\left(A / J, E_{q}^{I I}\right)=0 \text { pour } q=0, \ldots, \mu \text {. }
$$

Preuve: On raisonne par récurrence sur la longueur de $A / J$ en parcant du cas de longueur 1 donnć par le lemme 4.3. Le module $A / J$ possède une suite de composition dont les factcurs sont isomorphes à des $A / P_{i}$ où $P_{i}$ sont des idéaux bilatères maximaux de $A$. Soit $0 \subset H_{1} \subset H_{2} \subset \cdots \subset H_{s}=A / J$ une telle suite. Daprès le lemne, s'il existait un homomorphisme $f: A / J \rightarrow E_{q}^{\text {lf }}$ alors $f$ scrait nul sur $H_{\perp}$ et par hypothèse de récurrence serait nul sur $H_{s} / H_{1}$. Donc $f=0$.

4.4. Lemme. Chaque élément de $E_{p t}^{I}$ est annulé par un idéal coartinien de $A$.

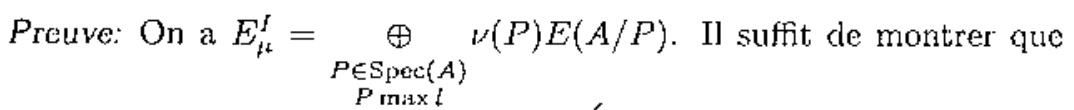

chaque élément de $E(A / P)$ est annulé paí un idéal co-artinien de $A$. Soit 
$x \in E(A / P) ; M=A / P$ est un $A$-module à gauche simple cssentiel dans $E(A / P)$; donc $M \subset A x$. Puisque $\operatorname{Ann}_{A}(M)=P \in \mathcal{F}$, il existe $J \in \mathcal{F}$ tel que J.Ax $\cap M \subseteq P M=(0)$. Comme $M$ est essentiel dans $A x$, on a $J A x=(0)$, c'est-à-dire $J x=0$ avec $J$ co-artinien.

Corollaire. On a $E_{\mu}^{I}=\underset{\mathcal{F}}{\lim _{\rightarrow}} \operatorname{Hom}_{A}\left(A / I, E_{i L}^{I}\right)$.

4.5. Le lemme suivant est adapté de la proposition 5.4 de [B.M].

Lemme. Soient $B$ un anneau premier, I un idéal bilatère de $B$ tel que $\operatorname{Hom}_{B}(B / I, B)=0$. Soit $0 \rightarrow B \rightarrow E_{0} \rightarrow E_{1} \rightarrow \ldots$ une résolution injective minimale du $B$-module ${ }_{B} B$. Soit $i \in \mathbb{N}$ tel que $\operatorname{Hom}_{B}\left(B / I, E_{l}\right) \neq 0$. Alors le grade du B-module à gauche $B / I$ est $\leq i$.

Prenve: Supposons que l'on ait $\operatorname{Ext}_{B}^{j}(B / I, B)=0$ pour toul $j \leq i$. Montrons par récurrence sur $k$ que $\operatorname{Hom}_{B}\left(B / I, E_{k}\right)$ cst nul pour tout $k \leq$ i. Puisque $\operatorname{Hom}_{B}(B / I, B)=0$, il en résulte que $\operatorname{Horm}_{B}\left(B / I, E_{01}\right)=0$. En effet si $x \in E_{0} \backslash\{0\}$ vérifie $l x=0$ on considère un élément $\alpha \in B$ tel que $0 \neq \alpha x \in B$ et on $I \alpha x=0$. D'où $\alpha x=0$, ce qui est la contradiction cherchée. Supposons $i>1$ et soit $0 \leq k<i$ tel que $\operatorname{Hom}_{B}\left(B / 1, E_{k}\right)$ soit mul. Montrons qu'il en est de même de $\operatorname{Hom}_{B}\left(B / I_{i} E_{k+1}\right)$. A partir des suites exactes

$$
E_{k} \stackrel{d_{k}}{\longrightarrow} E_{k+1}^{\prime} \stackrel{d_{k+1}}{\longrightarrow} E_{k+2}
$$

on obtient les suites exactes

$$
\operatorname{Hom}_{B}\left(B / I, E_{k}\right) \stackrel{\delta_{k}}{\longrightarrow} \operatorname{Hon}_{B}\left(B / I, E_{k+1}\right) \stackrel{\delta_{k+1}}{\longrightarrow} \operatorname{Hom}_{B}\left(B / I, E_{k+2}\right) .
$$

Comme $k+1 \leq i$, on a par hypothèse $\operatorname{Ext}_{B}^{k+1}(B / I, B)=0$; d'où l'on déduit ker $\delta_{k+1}=(0)$. Considérons les suites exactes

$$
0 \longrightarrow \mathrm{ker} d_{k+1} \longrightarrow E_{k+1} \stackrel{d_{k+1}}{\longrightarrow} E_{k+2}
$$

$0 \longrightarrow \operatorname{Hom}_{B}\left(B / I, \operatorname{ker} d_{k+1}\right) \longrightarrow$

$$
\longrightarrow \operatorname{Hom}_{B}\left(B / I, E_{k+1}\right) \stackrel{\delta_{k+1}}{\longrightarrow} \operatorname{Hom}_{B}\left(B / I, E_{k+2}\right) \text {. }
$$

On a $0=\operatorname{ker} \delta_{k+1}=\operatorname{Hom}_{B}\left(B / I, \operatorname{ker} \delta_{k+1}\right)$. D'où, puisque $E_{k+1}$ est l'eneloppe injective du $B$-module ker $\delta_{k+1}, 0=\operatorname{Hom}_{B}\left(B / I, E_{k+1}\right)$. 
4.6. Théorème. Soit $A$ un annean premier vérifiant les conditions $(A),(B)$ et $(C)$ et $E_{i}=$ le $i^{\text {ièrse }}$ terme de la résolution injective minimale

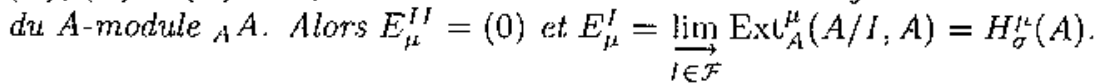

Preuve: D'après 4.2, $\underset{l \in \mathcal{F}}{\lim _{A}} \operatorname{Ext}_{A}^{\prime \prime}(A / I, A)=E$ est un $A$-module injectif et $T=\operatorname{Ext}_{A}(-, A)$ est naturellement ćquivalent à $\operatorname{Hom}_{A}(-, E)$ sur $\operatorname{Mod}_{A}^{\mathcal{F}}$.

On remarque d'abord que si $M$ est un $A$-module à gauche artinien alors $\operatorname{Hom}_{A}\left(M, E_{p}\right)=0$ si $p \leqq \mu$. En effect par un argument de récurrence sur la longueur de $M$ on se ramène aul cas où $M$ est simple. En effet si $\operatorname{Hom}_{A}\left(M, E_{p}\right)=0$ si $p \lessgtr \mu$ lorsque $M$ est simple alors $0 \rightarrow M^{\prime \prime} \rightarrow M \rightarrow$ $M^{\prime} \rightarrow 0, l\left(M^{\prime \prime}\right)<l(M)$ et $M^{\prime}$ simple, d'où

$$
\begin{aligned}
0 \longrightarrow \operatorname{Hom}_{A}\left(M^{\prime}, E_{p}\right)=0 \longrightarrow \operatorname{Hom}_{A}\left(M, E_{p}\right) \longrightarrow & \longrightarrow \\
& \longrightarrow \operatorname{Hon}_{A}\left(M^{\prime \prime}, E_{p}\right)=0 \longrightarrow 0 .
\end{aligned}
$$

Alors $M=A / J$ oi $J$ est un idéal bilatère co-artinien par (A). On a $j(M)=\mu \operatorname{car} \delta(M)=0$. Donc $\operatorname{Horn}_{A}(M, A)=0$. Il suffic d'appliquer 4.5. On a alors le résultat car si $\operatorname{Hom}_{A}\left(M, E_{i}\right)$ est non nul alors $j(M) \leq i$ done $i=\mu$.

Les foncteurs de $\operatorname{Mod}_{A}$ dans $A b, \operatorname{Ext}_{A}^{A t}(-, A)=T$ et $\operatorname{Hom}_{A}\left(-, E_{d t}\right) /$ $\operatorname{Im}\left(\operatorname{Hom}_{A}\left(-, E_{\mu-1}\right)\right)$ sont isomorphes. Puisque si $M \in \operatorname{Mod}_{A}^{\mathcal{F}}$ on a $\operatorname{Hom}_{A}\left(M, E_{t L-1}\right)=0$, sur la catégoric $\operatorname{Mod}_{A}^{\mathcal{F}}$ : les foncteurs $T$ et $\operatorname{Hom}_{A}\left(-, E_{\mu}\right)$ sont isomorphes et pour tout idléal bilatère $J$ co-artinien, les $A$-rnodules à gauche $T(A / J)$ et $\operatorname{Hom}_{A}\left(A / J, E_{\mu}\right)$ sont isomorphes.

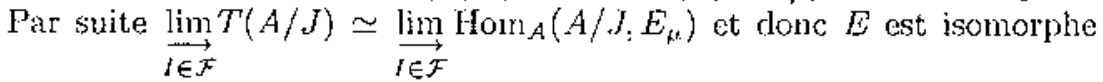

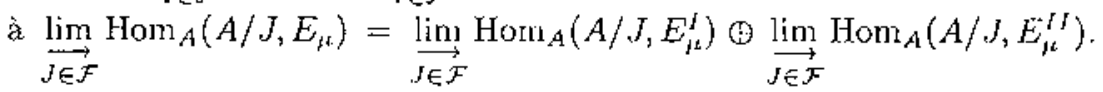
D'après 4.3 , on a que $E$ est isomorphe à $\lim _{J \in \mathcal{F}} \operatorname{Hom}_{A}\left(A / J, E_{\mu}^{I}\right)$ c'est-àdire à $E_{j k}^{I}$ d'après le corollaire 4.4 .

Montrons à présent que pour tout solis-module $M$ de type fini de $E_{\mu}^{I I}$ on a $\operatorname{Ext}_{A}^{\prime+}(M, A)=0$. Raisonnons par l'absurde et supposons que $Q=\operatorname{Ext}_{A}^{H}(M, A) \neq 0$. Puisque $\operatorname{Ext}_{A}^{i}\left(\operatorname{Ext}_{A}^{\mu}(M, A), A\right)=0$ si $i<\mu$, l'anneau $A$ étant Auslander-Gorenstein, il résulte du fait que $Q \neq 0$ que l'on a $\operatorname{Ext}_{A}^{\mu}(Q, A) \neq 0$. En effet si $\operatorname{Ext}_{A}^{\prime \prime}(Q, A)=0$ pour tout $\nu$ on aurait $\delta(Q)=-\infty$ et donc $Q=0$, d'après $(\mathrm{C})$. Alors Ext ${ }_{A}^{\prime t}\left(\operatorname{Ext}_{A}^{\prime t}(M, A), A\right)$ est un $A$-module à gauche non nul de longueur finie, puisque son $\delta$ esti nul. Il est isomorphe à $F^{\mu} M$, ce qui résulte du théorème $2.3(\mathrm{~b})$. Il existe 
donc un homomorphisme non nul $f: F^{t^{2}} M \rightarrow E_{t^{\prime}}^{H}$, ce qui contredit le corollaire 4.3.

Soit $E_{A}(A / I)$ une composante non multe de $E_{t h}^{H}$ : ì supposer qu'elle existe, ò $I$ est un idéal à gauclue de A. D'après ce qui précède on a $\operatorname{Ext}_{A}^{\prime \prime}(A / 1, A)=0 . S_{n-1} \stackrel{p_{k-1}}{\rightarrow} E_{\mu} \rightarrow 0$ est la demière Hèche de lic résolution injective minimale de $A$, l'application $\operatorname{Hom}_{A}\left(A / I, E_{\eta-1}\right) \rightarrow$ $\operatorname{Hom}_{A}\left(A / I, E_{\mu}\right)$ qui en résulte est surjective, puisque le coroyau $\operatorname{Ext}_{A}^{\prime 2}(A / I, A)$ est mul. Donc it existe un homomorphisme $f: A / I \rightarrow$ $E_{j t-1}$, tol que $p_{t k-1} \circ f$ soit l'identité sur $A / I$ et, par suite, $f$ est injectif. Comme ker $p_{j-1}$ est essentiel dans $E_{j t-1}$, on a alors Imf $\cap$ ker $p_{t-1} \neq 0$, ce qui contredit lo fait que $p_{k-1}$ of est l'identité sur $A / I$. Donc $E_{j t}^{H}=0$.

\section{References}

[A] AUSLANJER, M.; On the dimension of modules and algebras (III), Nagoya Math, \&. 9 (1955), 67-77.

[B-M] BAROU, G. EI MALlavin: M. P., Sur ba résolution injoctive minimale de l'algèbre enveloppante d'une algèbre de Lie résoluble. J. of Pure and Applied Alg. 37 (1985), 1 25.

[Ba] BASs, H., On the ubiquity of Corenstein rings, Math. Z, 82 (1963), 8-28.

[Bj1] B.öвк, J. E., "Rings of differential operators." North-Holland, 1979.

[Bj2] B.ı̈вk, .J. E., "The Auslonder condition on notherian rings," Séminaire Dubreil-Malliavin, 1987-88, L.N. in Math. 1404, Springer Verlag, 1989, pj. 137--173.

[Bj-Ek] Bıökк, J. E. E' Eksтü̈, E. K., "Filtered Auslander-Gorenstein rings," Colloque en lihonneur de .J. Dixmier; Birkhauser, 1990, pp. 424-448.

[Br] Brown, K., Ore sets in envelopping algebras, Compositio Math. 53 (1984): 347-367.

[Br-Le] Brown, K. IVT Levasseur, T., Cohomology of bimodules over enveloping algebras, Maht. Z. 189 (1985), 393-4l13.

[Ek] Eкsтӧм, E. K., "The Anstander Condition on graded and filtered neotherian rings," Sćminaire Dubroil-Malliaviu, 1987-88, L.N. in Math. 1404, Springer Verlag, 1989, pp. 220-245.

[F-G-R] FOSSLM, R., GRIFI'TH, P. AND RLITEN, 1.; "Thival extensions of Abelian categorics," L.N. in Math. 456, Springer Verlag; 1975. 
[He-Ve] Hendrux, B. and Verschoren, A., A note on compatibility and stability, Communications in Algebra 17(8) (1989), 1971-1979.

[Len] Lenagan, T., Gelfand Kirillov dimension and affine P.I. rings, Comm. Algebra 10, no. 1 (1982), 87-92.

[Le1] Levasseur, T., "Complexe bidualisant en algèbre non connnutative," Séminaire Dubreil-Malliavin, 1983-84, L.N. in Math. 1146, Springer Verlag, 1985, pp. 270-287.

[Lc2] Levasseur, T., Some properties of non commutative regular graded rings, (à paraître).

[Li-Hu] Ll, HusHl, Non commutative Zariskian rings, Pl. Doc. Thesis UIA, Antwerp (1989).

[Mal] MaLliavin, M. P., Modules sans torsion et modules injectives sur les algèbres de Lic résolubles, J. Algebra 83(I) (1983), 126-157.

[Ma2] Mallinvin, M. P., Représentations injectives d'algèbre de Lie résolubles: Comm. in Algebra 14(8) (1986), 1503-1513.

[Mc-Ro] MC Conneld, J. C. And Robson, J. C., "Non commutative noetherian rings," Wiley series in Pure and Applied Math., 1987.

[Re] Reiten, I., Trivial extensions and Gorenstein rings, Thesis Chicago Univ. (1971).

[Str] Strenström, B., "Rings of quotient," Springer, 1975.

[Ve] Verschoren, A., Compatibility and Stability, Notas de Matemática 3, Universidad de Murcia.

[Z] ZAKS, A., Injective dimension of semi-primary rings, J. of Algebra 13 (1969), 73-86.

10 Rue Sant Louis on I'Ile

75004 Paris

FRANCE

Primera versió rebuda el 14 d'Octubre de 1991, darrera versió rebuda el 18 de Novembre de 199 ] 